\title{
LOCAL MAGNETOHYDRODYNAMIC CHARACTERISTICS OF THE PLASMA STREAM GENERATED BY MPC
}

\author{
Tatyana N. Cherednychenko*, Igor E. Garkusha, \\ Vladimir V. Chebotarev, Dmytro G. Solyakov, Yuriy V. Petrov, \\ Maryna S. Ladygina, Dmytro V. Eliseev, Alexander A. Chuvilo \\ Plasma Physics Institute, National Science Centre "Kharkov Institute of Physics and Technology" Kharkov, Ukraine
}

* corresponding author: cherednichenko@kipt.kharkov.ua

\begin{abstract}
This paper investigates the spatial distributions of electrical current which flows inside the plasma stream generated by a magnetoplasma compressor (MPC). Two different modes of MPC operation with different gas supply scenarios have been applied in the experiments presented here. The first is the operation mode with a pulse injection of xenon into the interelectrode space, and the second is the operation mode with residual helium in the chamber and local injection of xenon directly into the compression zone. The maximum value of the electric current observed outside the MPC channel is $15 \div 20 \%$ of the total discharge current. Electric current vortices were discovered in the plasma stream. The amplitude of the current in the vortices reaches $50 \%$ of the total discharge current. The maximum EUV radiation power was measured in the mode of MPC operation with local xenon injection. Power in the wave range $12.2 \div 15.8 \mathrm{~nm}$ achieves up to $16 \div 18 \mathrm{~kW}$.
\end{abstract}

KEYWORDS: magnetoplasma compressor, toroidal current vortices, plasma discharge.

\section{INTRODUCTION}

This paper presents an investigation of the magnetohydrodynamic characteristics of the plasma stream generated by a magnetoplasma compressor (MPC). The distributions of the electric currents in the plasma stream were measured for different operating regimes. The maximum density of the plasma stream in the compression zone is about $10^{18} \mathrm{~cm}^{-3}$, and the average electron temperature along a line of view is $\sim 5 \div 7 \mathrm{eV}$, and the velocity of the plasma stream at the output of MPC is $\sim 10^{7} \mathrm{~cm} / \mathrm{sec}$. The spatial distributions of the electric current in the plasma stream were measured and the spatial distributions of electromagnetic forces were investigated.

\section{EXPERIMENTAL SETUP}

The experiments were carried out in an MPC with a compact geometry [4, 1. A general view of the MPC facility is presented in Fig. 1] The MPC channel is formed by coaxial copper electrodes. The outer electrode is a semitransparent multi-rod anode with output diameter $80 \mathrm{~mm}$, and the inner electrode is a solid cathode with output diameter $40 \mathrm{~mm}$. The power supply system for the MPC discharge is a bank of condensers with total capacity $(90 \mu \mathrm{F})$ and voltage up to $25 \mathrm{kV}$. The maximum value of the discharge current is $500 \mathrm{kA}$ and the duration of the half period is $10 \mu \mathrm{s}$.

Two different modes of MPC operation were investigated [2]. The first mode operates in the residual gas (helium) with pressure $2 \div 10$ Torr. The second mode

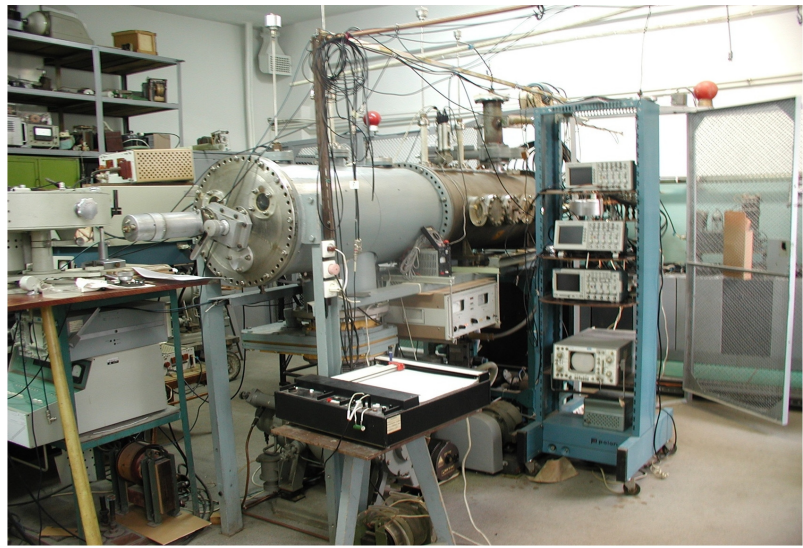

Figure 1. General view of MPC experimental setup.

is operation with the residual gas and additional local injection of xenon directly into the compression zone (Fig. 2).

The Rogowski coil is applied for the discharge current measurements. A high voltage divider is used for the discharge voltage measurements. Numbers of local movable magnetic probes are used for an investigation of the spatial distributions of the electric current in the plasma stream. The electron density was estimated from the Stark broadening of the spectral lines. The plasma stream velocity was measured by the timeof-flight method of the plasma stream between two electric probes. Various types of AXUV diodes [3] are used for an analysis of the plasma stream radiation in the EUV wavelength range of $5 \div 80 \mathrm{~nm}$. 


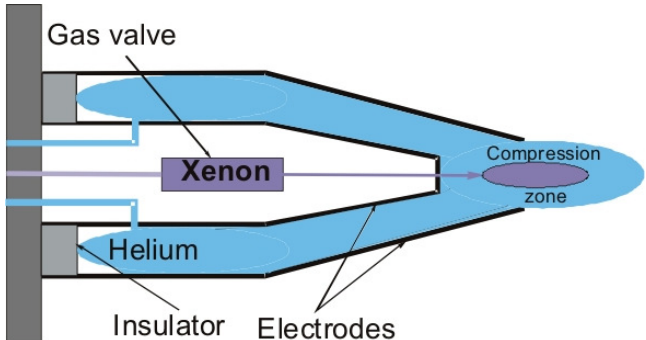

Figure 2. The mode of MPC operations with residual helium and additional local injection of xenon directly into the compression zone.

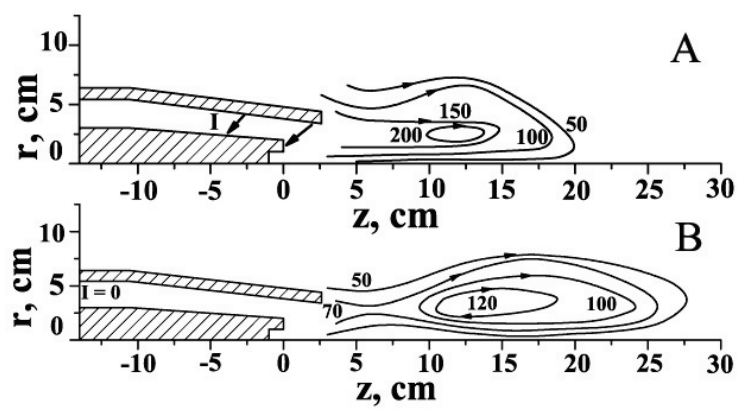

FIgURE 3. The spatial distributions of the electric current in the plasma stream for the first operation mode; a) $6 \mu \mathrm{s}$, b) $10 \mu \mathrm{s}$.

\section{EXPERIMENTAL RESUlTS}

The spatial distributions of the electric current that were measured experimentally in the plasma stream for two MPC modes of operation are presented in Figs. 3 and 4 . It is evident that a part of the discharge current flows into the plasma stream generated by MPC for both operation modes. The maximum value of the electric current in the plasma stream outside of the MPC channel is not more than $15 \%$ of the total discharge current. Figure 3 shows a very interesting effect when the magnetic field is pushed out from the compression zone at a distance of $5 \div 10 \mathrm{~cm}$ from the output of the MPC for the first regime. The current vortex is formed at a distance of $10 \div 25 \mathrm{~cm}$. In cases when the MPC is changed from the operation regime to the mode with residual gas and additional pulse xenon injection directly into the compression zone, a vortex of electric current also forms some distance away, and displacement of magnetic field is also observed. However, the radial dimension of the displacement zone reduces and the length of the area without a magnetic field increases in comparison with the previous MPC regime.

Figure 5 shows the spatial distribution of the Lorentz force. The helium pressure is 2 Torr and the time is $t=10 \mu \mathrm{s}$. From this figure we can clearly see the areas where the plasma stream is decelerated and where the plasma stream is accelerated. We can see the area with a compression plasma stream and the area where the plasma stream moves in the direction of the wall of the vacuum chamber. According to

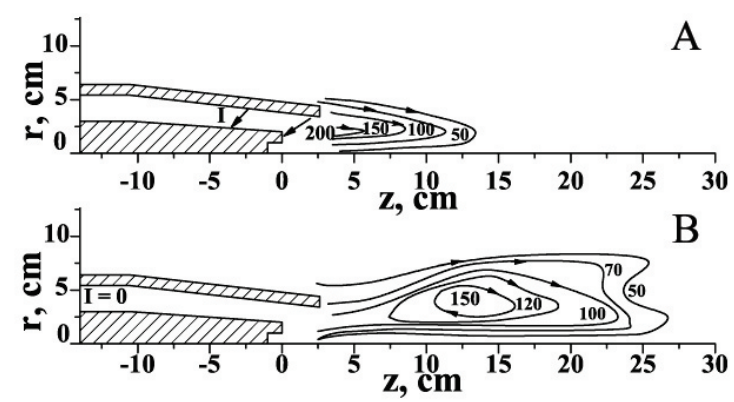

Figure 4. The spatial distributions of the electric current in plasma stream for the second operation mode; a) $6 \mu \mathrm{s}$, b) $10 \mu \mathrm{s}$.

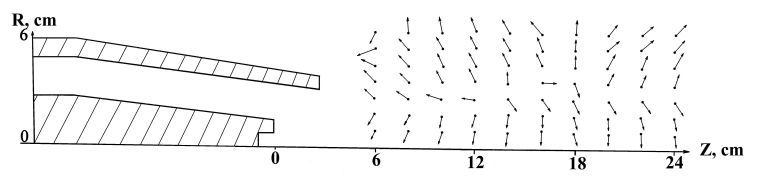

Figure 5. The spatial distribution of the Lorentz force, pressure of 2 Torr, time $t=10 \mu \mathrm{s}$.

the Bernoulli equation, the total energy of the plasma stream which consists of the kinetic energy, the thermal energy and the energy of the magnetic field, has a constant value

$$
\frac{v^{2}}{2}+\int \frac{\mathrm{d} p}{\rho}+\frac{H^{2}}{4 \pi \rho}=\text { const. }=U .
$$

In the MPC channel, the plasma stream has kinetic energy only. The kinetic energy of the plasma stream converts to thermal energy in the compression zone, and after that the thermal energy converts to the kinetic energy of the plasma stream and the energy of the magnetic field.

Figure 6 presents the dependencies of the longitudinal component of the electromagnetic force, the magnetic pressure and the intensity of the radiation of the three Xe spectral lines $(362.4 \mathrm{~nm}, 378.1 \mathrm{~nm}$ and $395 \mathrm{~nm}$ ).

This figure shows the radiation of the xenon lines in the area of maximum stream deceleration only. When the plasma stream passes through the compression zone and acceleration begins, there is no Xe radiation. At the same time, an electric current vortex is generated in the area where the plasma stream is accelerated. Thus the initial kinetic energy of the plasma stream transforms to thermal energy in the compression zone, where the plasma stream is compressed and heated. When the plasma stream passes through the compression zone, the thermal energy converts to kinetic energy and to the energy of the magnetic field. And as a result, a toroidal vortex of electric current generates in the plasma stream. 


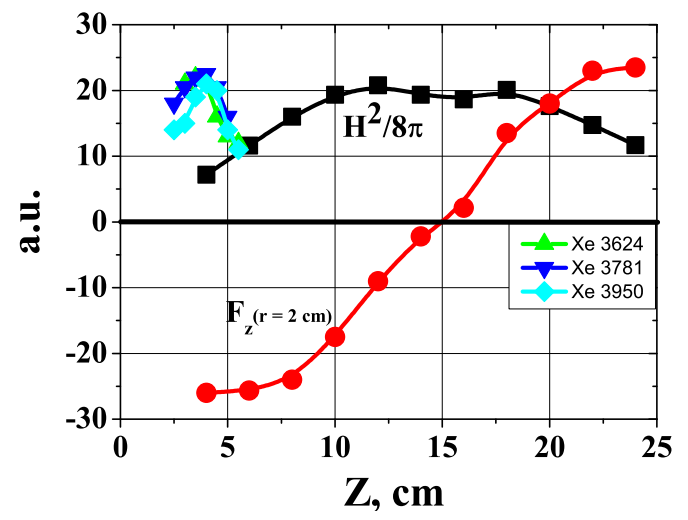

Figure 6. Distributions of the longitudinal component of the electromagnetic force, the magnetic pressure $\left(H^{2} / 8 \pi\right)$ and the emission intensity of the three spectral lines of xenon along the $Z$ axis.

\section{Summary AND CONClusions}

Two modes of MPC operation have been investigated. Plasma streams with density in the compression zone up to $(1 \div 2) \times 10^{18} \mathrm{~cm}^{-3}$ are obtained. The electron temperature, estimated on the basis of the pressure balance equation, is $60 \div 100 \mathrm{eV}$.

The spatial distribution of the electrical current in the plasma stream for two modes of MPC operation has been investigated. It has been shown that the maximum value of the electrical current that flows in the plasma stream generated by MPC is not more than $15 \div 20 \%$ of the discharge current in the MPC channel. The toroidal vortex of the electric current with the value of the current up to $50 \%$ of the discharge current was observed in the plasma stream. The displacement of the magnetic field from the com- pression zone has been discovered.

The spatial distributions of the electromagnetic force in the plasma stream are calculated. It is shown that the plasma stream is decelerated in the compression zone. The kinetic energy of the plasma stream converts to thermal energy in the compression zone. The density of the plasma stream increases up to $(1 \div 2) \times 10^{18} \mathrm{~cm}^{-3}$ and the plasma temperature reaches $60 \div 100 \mathrm{eV}$.

The radiation of the xenon spectral lines in different wavelength ranges has been observed from the compression zone. In the optimal mode of MPC operation, with local xenon injection directly into the compression zone, the maximum value of the radiation energy in the waverange of $12.2 \div 15.8 \mathrm{~nm}$ is $(5 \div 6) \times 10^{-2} \mathrm{~J}$, and the maximum value of the power is $16 \div 18 \mathrm{~kW}$.

\section{REFERENCES}

[1] V. V. Chebotarev, I. E. Garkusha, et al. Dynamics of nitrogen and xenon plasma streams generated by MPC device. Problem of Atomic Science and Technology 13:104-106, 2007.

[2] I. E. Garkusha, et al. Discharge characteristics and dynamics of compressive plasma streams generated by a compact magnetoplasma compressor. Plasma Physics Reports 37(11):948-954, 2011.

[3] Yu. V. Petrov, I. E. Garkusha, A. Hassanein, et al. Diagnostic system for EUV radiation measurements from dense xenon plasma generated by MPC. Problem of Atomic Science and Technology 17:185-187, 2011.

[4] V. V. Chebotarev V.V., I. E. Garkusha, M. S. Ladygina, et al. Investigation of pinching discharges in MPC device operating with nitrogen and xenon gases. Czechoslovak: Journal of Physics 56(S2):B335-B341, 2006. 